\title{
Successful Explantation of a Ball and Cage Mitral Valve Prosthesis 48 Years After Initial Implantation
}

\author{
Kenza Rahmouni, MD, ${ }^{a}$ Justendra Naidu, MD, ${ }^{b}$ Neil Pearce, MD, ${ }^{c}$ and Vincent Chan, MD, $\mathrm{MPH}^{\mathrm{a}}$ \\ ${ }^{a}$ Division of Cardiac Surgery, University of Ottawa Heart Institute, Ottawa, Ontario, Canada \\ ${ }^{b}$ Department of Anesthesiology, St. Paul's Hospital, University of British Columbia, Vancouver, British Columbia, Canada \\ ${ }^{c}$ Division of Cardiology, Memorial University of Newfoundland, St. John's, Newfoundland and Labrador, Canada
}

\begin{abstract}
A 58-year-old female had undergone previous mechanical mitral replacement with a Starr-Edwards ball and cage valve (Edwards Lifesciences, Irvine, CA) at 11 years of age for rheumatic disease. The valve functioned well until pannus resulted in prosthetic valve stenosis with class IV dyspnea and pulmonary hypertension. She underwent reoperative mitral replacement with an On-X mechanical mitral prosthesis (Cryolife, Kennesaw, GA) 48 years after initial implantation. To our knowledge, this case represents the longest known implant period of a ball and cage mitral valve in the literature. This presentation highlights the durability of this historic prosthesis, along with the role of reoperative surgery decades after initial implantation.
\end{abstract}

A 58-year-old female previously had undergone mechanical mitral valve replacement with a Starr-Edwards ball and cage prosthesis (Edwards Lifesciences, Irvine, CA) at 11 years of age for rheumatic mitral stenosis. She suffered a large embolic stroke with hemiparesis when she was 21 years old. At that time, she was taking only low-dose aspirin, but she was started on warfarin after her stroke. Her neurologic symptoms completely resolved shortly after her cerebrovascular accident, and she remained functionally well until 2017, when she developed New York Heart Association class IV dyspnea refractory to medical therapy. She had chronic atrial flutter, and transthoracic echocardiography confirmed severe mitral stenosis with gradients of 17 $\mathrm{mm} \mathrm{Hg}$ (mean) and $35 \mathrm{~mm} \mathrm{Hg}$ (peak), with normal biventricular function and moderate pulmonary hypertension.

Forty-eight years after her first cardiac surgery, the patient underwent redo sternotomy, explantation of the ball and cage

Received for publication November 1, 2021. Accepted November 17, 2021.

Ethics Statement: The patient consented to this publication.

Corresponding author: Dr Vincent Chan, Division of Cardiac Surgery, University of Ottawa Heart Institute, 40 Ruskin St, Ottawa, Ontario K1Y 4W7, Canada. Tel.: +1-613-696-7294; fax: +1-613-696-7302.

E-mail: vchan@ottawaheart.ca

See page 346 for disclosure information.

\section{RÉSUMÉ}

Une femme de 58 ans avait subi un remplacement mécanique d'une valve mitrale par une valve à bille Starr-Edwards alors (Edwards Lifesciences, Irvine, CA) qu'elle avait 11 ans par suite d'une maladie rhumatismale. La valve avait bien fonctionné jusqu'à ce que la formation de pannus entraîne une sténose de celle-ci s'accompagnant de dyspnée de classe IV et d'hypertension pulmonaire. La femme a subi une intervention chirurgicale visant à remplacer la valve sténosée par une valve mécanique On-X (Cryolife, Kennesaw, GA) 48 ans après l'implantation initiale. À notre connaissance, il s'agit de la plus longue période d'implantation connue d'une valve mécanique mitrale à bille Starr-Edwards rapportée dans la littérature. Le présent article met en évidence la durabilité de cette prothèse historique, ainsi que le rôle de la chirurgie réopératoire des décennies après l'implantation initiale.

valve, and mitral valve replacement with a \#25 On-X mechanical prosthesis (CryoLife, Kennesaw, GA). The patient selected a new type of mechanical prosthesis because she wanted to minimize her risk of needing further reoperation and was comfortable managing anticoagulation therapy with a vitamin-K antagonist. Intraoperatively, the ball and cage prosthesis was covered in dense calcified pannus, which was also visible on transesophageal echocardiography (Fig. 1). The prosthesis was carefully extracted from the mitral annulus, and all the calcific debris was removed. The new prosthesis functioned well, with a mean gradient of $2 \mathrm{~mm} \mathrm{Hg}$ on transesophageal echocardiography. During her recovery, she developed symptomatic bradycardia with a persistent junctional heart rhythm while in the hospital and required singlelead pacemaker implantation. She remains well, without functional limitations, 10 months after her surgery.

\section{Discussion}

The Starr-Edwards ball and cage mitral valve prosthesis was designed in 1960 by Albert Starr and Miles Edwards. ${ }^{1,2}$ The design relies on the random rotation of a ball contained within a cage, allowing it to wear evenly. ${ }^{2}$ This valve was extremely durable, and its design was nearly unchanged for over 40 years. $^{2}$ 


\section{Novel Teaching Points}

- Although the ball and cage mitral valve prosthesis is extremely durable, its use was discontinued in 2007 due to unacceptable rates of thromboembolic complications.

- Because of the durability of this older-generation mechanical prosthesis, patients may present with a need for reoperation several decades after initial implantation.

- Therefore, even though ball and cage prostheses are no longer available, clinicians should maintain knowledge of these valves and the role of late reoperative surgery.

Despite the early promising results, ${ }^{1}$ patients who received a Starr-Edwards valve had increased thrombotic risk, compared with patients who received a tissue prosthesis. ${ }^{2}$ Interesting to note is that Ikizler and colleagues reported the case of a patient who, despite not taking anticoagulant therapy, remained free of thromboembolic events, intracardiac thrombus, and prosthetic valve dysfunction 34 years after the implantation of an aortic Starr-Edwards prosthesis. ${ }^{3}$ However, this case likely represents an exception to the more usual course, as mechanical valves are well known for their high risk of thromboembolic complications, in both the aortic and mitral positions. ${ }^{4}$ In fact, our patient suffered a large thromboembolic stroke at age 21 years while on only aspirin. Even with the introduction of anticoagulation therapy, rates of thromboembolism remained significant in patients with a ball and cage prosthesis, leading to the discontinuation of use of the valve in $2007 .^{2}$

Between 1960 and 2007, over half a million Starr-Edwards valves were implanted globally. Therefore, these valves remain prevalent in the population, even though they are no longer commercially available. The explantation of a ball and cage prostheses in the mitral position, between 31 to 43.3 years after
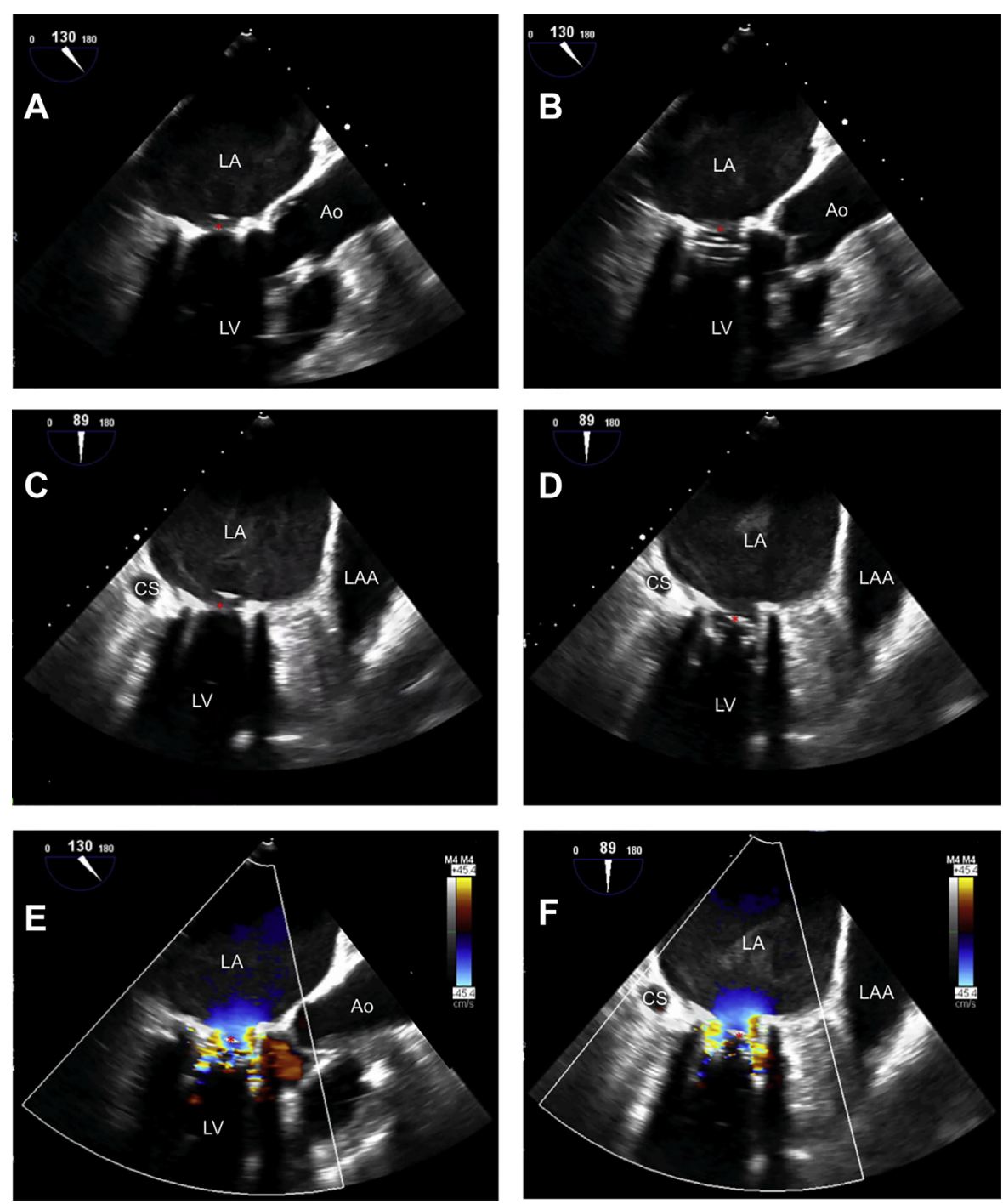

Figure 1. Transesophageal echocardiogram showing the ball and cage mitral valve prosthesis (red asterisk). (A, B) Mid-esophageal long-axis view showing the valve in (A) systole and (B) diastole. (C, D) Mid-esophageal commissural view showing the valve in (C) systole and (D) diastole. (E, F) Mid-esophageal (E) long-axis and (F) commissural views in diastole showing flow acceleration through the prosthesis. Ao, aorta; CS, coronary sinus; LA, left atrium; LAA, left atrial appendage; LV, left ventricle. 
initial implantation, has been previously reported. ${ }^{2,5,6}$ For instance, Head et al. ${ }^{5}$ described a case of a mitral Smeloff-Cutter ball-caged valve (Cutter Laboratories, Berkeley, CA) that failed more than 4 decades after implantation, owing to lipid absorption, which resulted in ball motion irregularity and poor valve sealing. In fact, in a ball and cage valve prostheses, the 2 most common mechanisms of late valve dysfunction are ball motion variance, caused by lipid absorption, and pannus formation, as occurred in our patient. ${ }^{5}$

Contemporary mechanical valves may offer better longevity than the historic ball and cage valve, with linearized annual rates of major hemorrhage and thromboembolism of approximately $1.2 \%$ and $0.7 \%$, respectively. In addition, although newer oral anticoagulation agents have not yet been shown to be safe for use with mechanical prostheses, the Prospective Randomized On-X Anticoagulation Clinical Trial (PROACT) Xa trial, comparing apixaban to warfarin anticoagulation in aortic On-X prostheses (CryoLife), is currently underway. ${ }^{8}$ This trial may constitute a first step toward improving the quality of life of patients with mechanical prostheses, namely by ending the need for both vitamin- $\mathrm{K}$ antagonist anticoagulation and close international normalized ratio monitoring. Nonetheless, vitamin-K antagonist is universally recognized as the gold-standard therapy for anticoagulation in mechanical valves, and changing this practice will likely be challenging, especially for mitral prostheses.

In summary, we report the case of a patient who underwent successful explantation of a Starr-Edwards mitral valve prosthesis 48 years after implantation. To our knowledge, this is the longest interval between implantation and explantation of a ball and cage mitral valve reported in the literature. This case highlights the durability of this historic prosthesis, along with the role of reoperative surgery late after initial implantation.

\section{Acknowledgements}

We thank the patient for consenting to this publication.

\section{Funding Sources}

The authors have no funding sources to declare.

\section{Disclosures}

The authors have no conflicts of interest to disclose.

\section{References}

1. Starr A, Edwards ML. Mitral replacement: clinical experience with a ballvalve prosthesis. Ann Surg 1961;154:726-40.

2. Battaglia F, Mielniczuk L, Dupuis JY, Chan V. Explant of a ball and cage valve 42 years after initial implant. J Thorac Cardiovasc Surg 2018;155: e147-8.

3. Ikizler M, Birdane A, Sevin B. An old friend is still at work: 34-year-old well functioning Starr-Edwards aortic prosthesis without anticoagulation. Int J Cardiol 2007;116:e1-3.

4. Goldstone AB, Chiu P, Baiocchi M, et al. Mechanical or biologic prostheses for aortic-valve and mitral-valve replacement. N Engl J Med 2017;377:1847-57.

5. Head SJ, Ko J, Singh R, Roberts WC, Mack MJ. 43.3-year durability of a Smeloff-Cutter ball-caged mitral valve. Ann Thorac Surg 2011;91:606-8.

6. Goshima M, Shiono M, Yamamoto T, et al. [Reoperation for a StarrEdwards ball valve prosthesis implanted in mitral position 31 years ago]. Kyobu Geka 2003;56:535-40 [Article in Japanese].

7. Chan V, Jamieson WR, Lam BK, et al. Influence of the On-X mechanical prosthesis on intermediate-term major thromboembolism and hemorrhage: a prospective multicenter study. J Thorac Cardiovasc Surg 2010;140:1053. 8.e2.

8. Jawitz OK, Wang TY, Lopes RD, et al. Rationale and design of PROACT Xa: a randomized, multicenter, open-label, clinical trial to evaluate the efficacy and safety of apixaban versus warfarin in patients with a mechanical On-X aortic heart valve. Am Heart J 2020;227:91-9. 